This PDF is a simplified version of the original article published in Internet Archaeology. All links also go to the online version.

Please cite this as: Jantos, S. and Sommer, C.S. 2021 Digital Archiving and Process Management in the Bavarian State Department of Monuments and Sites, Internet Archaeology 58.

https://doi.org/10.11141/ia.58.4

\title{
Digital Archiving and Process Management in the Bavarian State Department of Monuments and Sites
}

\author{
Silke Jantos and C. Sebastian Sommer
}

\section{Summary}

Over the last two decades, technical progress for archaeology within the Bavarian State Department of Monuments and Sites (BLfD) underwent rapid development. It began with the establishment of an information system (FIS) where the workflow of the Department of Archaeological Heritage is mapped. The next step consisted of standardisation of data capture for all institutions and companies undertaking excavation. In response, a homogeneous data model was developed and established through the application ExcaBook. In order to guarantee this solution would be used widely, an Importer was created to import data from other databases or applications. Hereafter it is also planned to process data from restoration and conservation projects, using a similar approach in order to work towards improving data exchange between all related sciences.

\section{Need for Digital Archiving}

The need for digital archiving has been recognised within the Department of Archaeological Heritage Management of the Bavarian State Department of Monuments and Sites (Bayerisches Landesamt für Denkmalpflege - BLfD) for many years. Legislation concerning heritage in Bavaria was passed in 1973 (Bayerisches Denkmalschutzgesetz - BayDSchG), requiring interventions taking place on or close to actual or predicted archaeological heritage sites to acquire permission prior to undertaking any work penetrating the ground.

Bavaria contrasts with other Federal States in Germany and neighbouring countries because the vast majority of excavations are conducted by commercial excavation 
companies, rather than by the Bavarian State Department of Monuments and Sites (BLfD). Therefore, the necessity of creating formal guidelines concerning excavation documentation was recognised. Nevertheless, the data being delivered by excavation companies are still rather diverse, as the guidelines still give latitude to the individual data creator. Even if different companies use similar terminology to describe features and finds, their content is often different and not necessarily comparable, because data types vary, for example. Additionally, the software employed differs from company to company. Hence databases with different data models, MS Excel spreadsheets, other lists or even handwritten sheets can be used, because there is currently no legislation that pertains to digital archiving.

Since excavations represent a controlled destruction of the archaeological heritage, a thorough and complete capture of the data should be required. The advent of digital data should allow us to take advantage of the developments of the digital age and to start collecting data in standardised and interoperable ways.

\section{Workflow Reproduced by the Information System (FIS) of the Bavarian State Department of Monuments and Sites (BLfD)}

In the early 2000s, the BLfD started to develop a specialised information system (FIS) to display the workflow from the planning phase through the excavation phase to postexcavation results including the possibility to analyse the captured data, all geolocated. FIS is a proprietary web-based expert information system using a Postgres database. The different underlying maps are provided via external servers, regulated through cooperation with the land surveying office. From 2007 onwards, the relevant workflow and the registration of excavations in Bavaria was executed exclusively within FIS. At the same time, digitisation of geometry and metadata of all former excavations in Bavaria commenced.

The process of gaining permission for groundworks on or near to known archaeological sites often starts with rural or urban land-use planning that will result in development. The different planning steps are recorded in FIS with all relevant correspondence, creating a record for all users who may be involved in present and/or future procedures.

Where archaeological sites are concerned, the detailed planning as well as any subsequent intervention, may result in one or more activities or excavations, and a work order is placed with an excavation company by the owner. Concerning the excavations conducted by excavation companies, metadata such as 'preceding planning, start and completion date of the excavation, name of the company, geometry, etc.' are captured (Figure 1). 


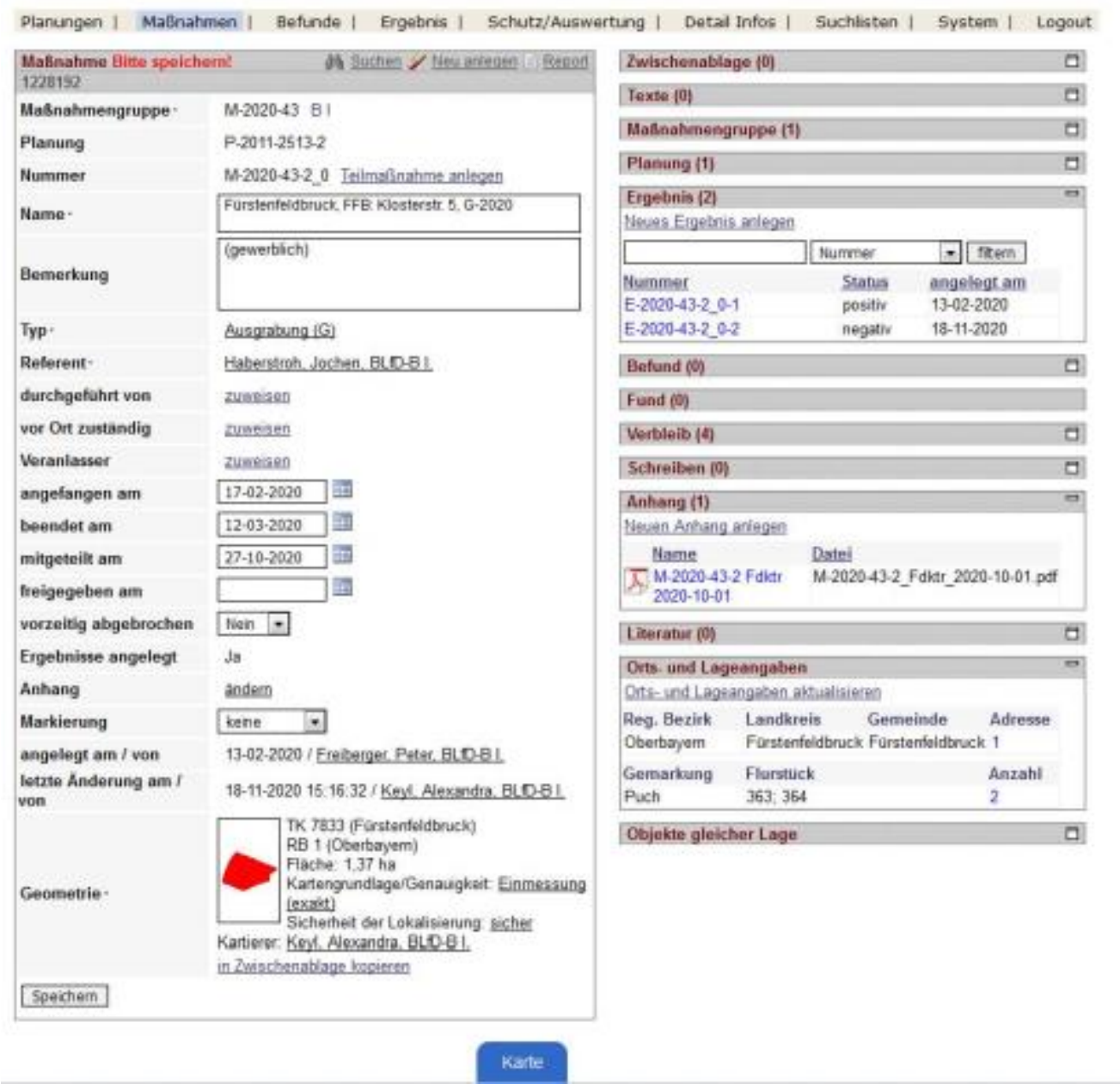

Figure 1: Example of metadata for an excavation recorded in FIS

After finishing an excavation, which may yield one or more positive or negative results and include information relating to different time periods or artefact types, the latter are registered with suitable attributes and geometry in FIS. The results recorded in FIS may affect the designation of old or new archaeological sites, and eventually subsequent planning procedures.

Additionally, the disposition of finds is registered. This is of major importance, since Bavaria is the only Federal State in Germany without so-called treasure trove legislation or definite regulation concerning the ownership of archaeological finds. Therefore, potential owners of archaeological artefacts must be identified, prior to permanent storage of finds within different physical archives.

Hence, it is now possible to get a broad overview of archaeological interventions across Bavaria, as well as evaluating the data from individual sites (Figure 2). 


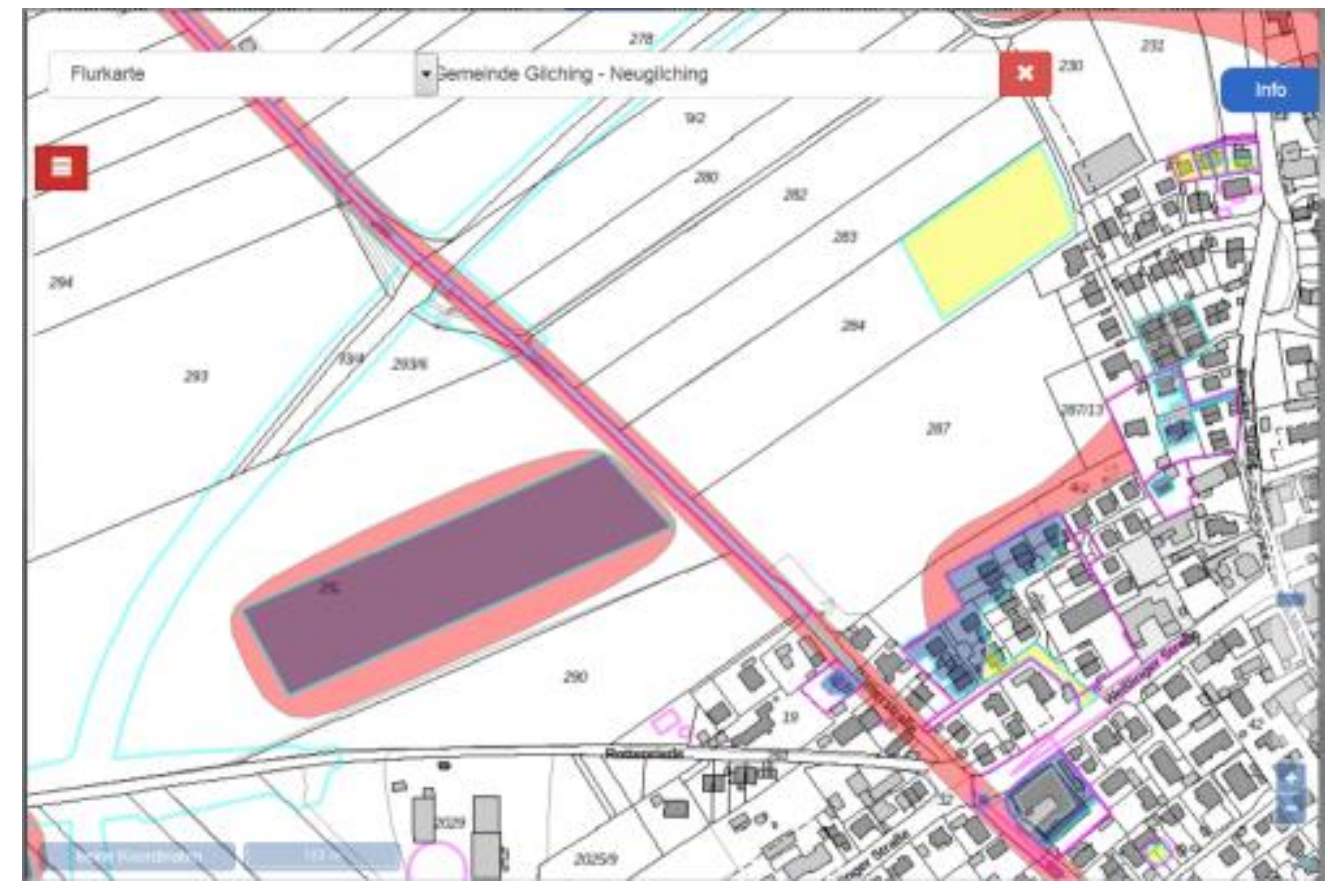

Figure 2: Mapped heritage sites (red), excavation measures (blue or yellow), planning (pink border) and other activities within the FIS

Although FIS is a suitable instrument for the administration of metadata and workflows, the data obtained through excavation, such as the listing and descriptions of features, finds, photos, diaries etc. are handled in various ways and without adherence to standards, although formal guidelines are given. This led to the need to develop a database to gather homogeneous data from excavations within a database, in order to facilitate data retrieval and comparison.

\section{Development/Use of ExcaBook}

In 2011, Daniel Kaltenthaler and Johannes Lohrer (Ludwig-Maximilians-Universität Munich - LMU) developed the framework XBook as an extraction of OssoBook (database of the ArchaeoBioCenter of LMU for the standardised collection of zooarchaeological data). Thereby the basic architecture was created to provide a generic solution to support administration of the data, as well as the work of scientists in archaeology-related disciplines (Kaltenthaler 2018; Kaltenthaler et al. 2020).

In 2013 the ARCHAEOBioCenter of the LMU Munich approached the BLfD with the requirement to connect archaeology-related archives with the original excavation data gathered from companies. The first problem to be encountered was that no uniform database currently existed.

Kaltenthaler and Lohrer therefore began to develop an archaeological excavation database, with a data model adapted to the requirements of archaeological heritage management (data including features, finds, photos, etc.) (Figure 3). This application was called ExcaBook and is now used to collect data from different excavation companies and projects. A major advantage of ExcaBook is the ability to synchronise projects from different computers or servers, which enables co-workers within a project to capture and combine data - e.g. field data and office data - at the same time. 


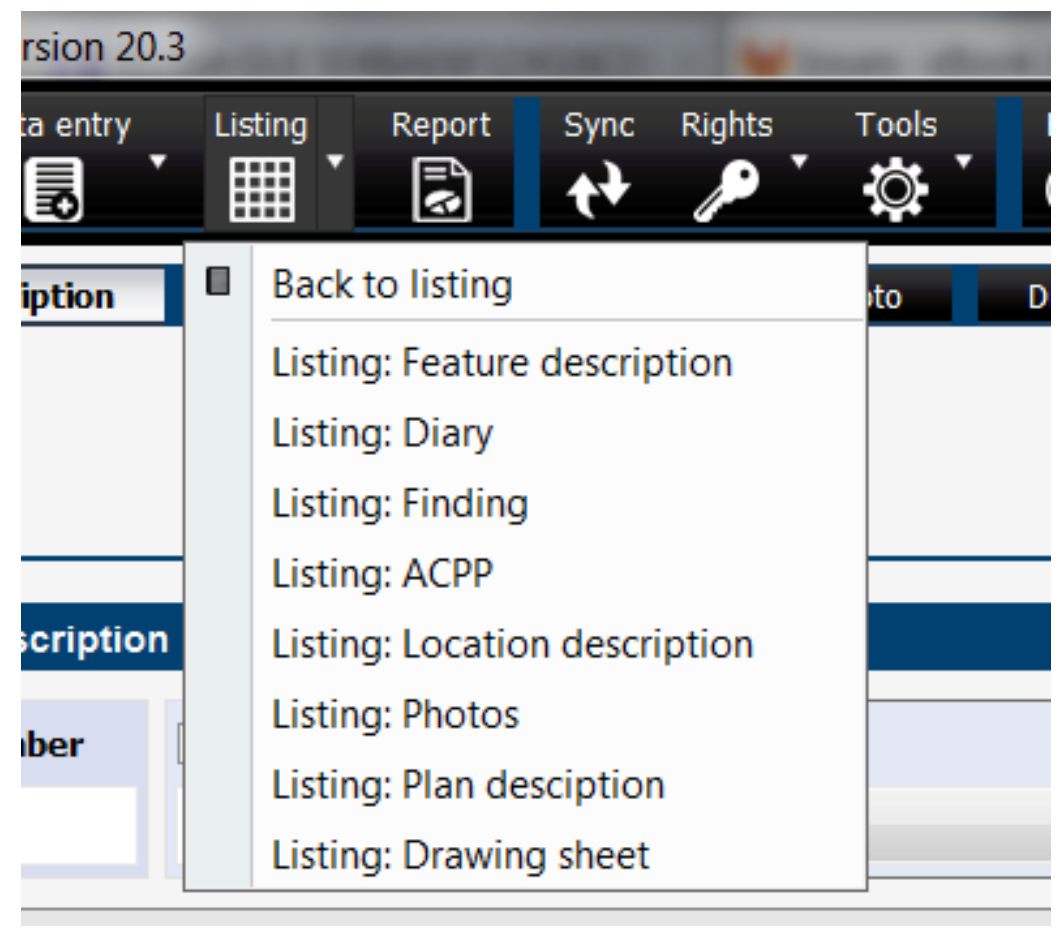

Figure 3: Tables within ExcaBook

This web-based data exchange improves scientific evaluation and retrieval of crucial data, and furthermore ensures digital data is safely stored in a data processing service centre, on a managed server with 24-hour access.

Furthermore, ExcaBook offers the ability to capture and manage homogeneous data for the first time, since excavation companies in Bavaria deliver data using the same scheme. Data can be exported (CSV, XLSX), and hard-copy reports can be generated by the database application. The authorisation tool makes sure that only permitted users have access to it.

The development of ExcaBook is financed by the BLfD, and both academic and professional users have free access to the database application.

ExcaBook is a nearly self-explanatory and easy to handle tool that simplifies documentation work (Figure 4). Hence, users automatically implement the actual formal guidelines given by the BLfD, using the report functionality. To further facilitate work within a database by multiple co-workers on a single project, a tool to discover data inconsistencies was included. In addition, it allows consistent data collection without any redundancy because, according to the data model, all objects are related to each other. In using and always updating the same database, long-term usefulness of the data is guaranteed. The development of ExcaBook is continuously in progress, and the experiences of users are incorporated to improve the software. 


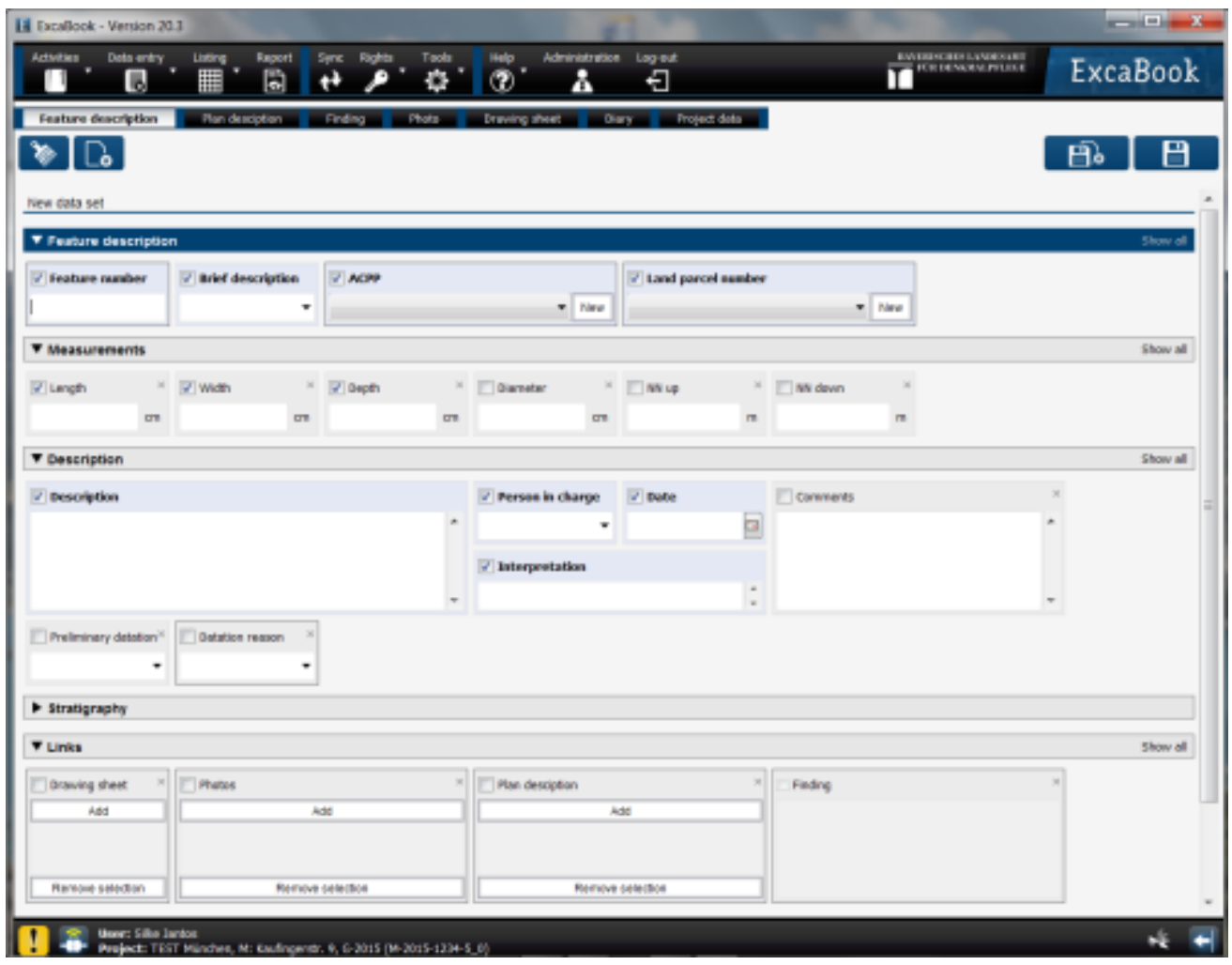

Figure 4: The table 'Feature' and its attributes within ExcaBook

The technical requirements for this server-client application is based on a MySQLdatabase PostgreSQL) and a current version of Java. With this reliable server-client infrastructure, it is also possible to synchronise and back-up data. It is also important to have a managed architecture that can be accessed and used from everywhere without any restrictions and multiple users can create, edit, and share their data. During Covid lockdowns this meant that it was also possible to administer the database or capture data from the home office. Another advantage is the ability to work online or offline. For installation and administrative work users must be online, and their changes will be directly stored on a managed server situated in the LRZ (Leibniz Supercomputing Centre). Crucial for the usage of ExcaBook on different sites is the possibility of offline operation. Synchronisation with the server can occur later when an internet connection is available.

\section{Data Import from Different Databases into ExcaBook}

To achieve better acceptance by the user, a data interface (importer) is in development to enable companies with an independent database solution to continue to use their own applications and also render standardised data. As it is not possible to make use of a particular database/software obligatory, the companies that use their own databases must be given the ability to migrate their data into ExcaBook. As soon as the importer is completed, it will be possible for all companies to store their data within ExcaBook. 
This importer, developed by Cesonia $\mathrm{GmbH}$, will be accessible from everywhere with an individual login, and offers a way to map data from different databases or formats (MySQL, Access, Excel, CSV) as well as import them in suitable format into ExcaBook.

With this tool we are on the right path to achieve the target of capturing and storing all data related to Bavarian sites within ExcaBook, either by collecting the data directly in ExcaBook or migrating it using the importer.

The final step after the completion of an archaeological project and the storage of the data in ExcaBook, is the import of the data from ExcaBook into the information system (FIS), where it is embedded into the planning workflow and e.g. the geo-referencing of the project will take place.

\section{Perspective}

Meanwhile, the need for a database that answers the needs of archaeological conservation and restoration workflows has been recognised. There are ideas about creating another 'Book' as an add-on for restoration and conservation of finds to be embedded in the environment of FIS and ExcaBook.

Another big project brought about by the development of ExcaBook is a digital find-ID that identifies finds e.g. that are stored at the ASM (State Archaeological Collection Munich) with the finds data recorded at the excavation.

Within the framework of the Archaeological Geo-Data-Network (an interdisciplinary project of the ARCHAEOBioCenter at the LMU Munich) an exchange of databases of archaeological related sciences like AnthroBook (a standardised collection of morphological data within Anthropology), ArchaeoBook (an inventory database for archaeological finds) and OssoBook (scientific database for the standardised collection of zooarchaeological data) is planned. This scenario is, of course, highly dependent on all participants agreeing to use the databases (Figure 5). 


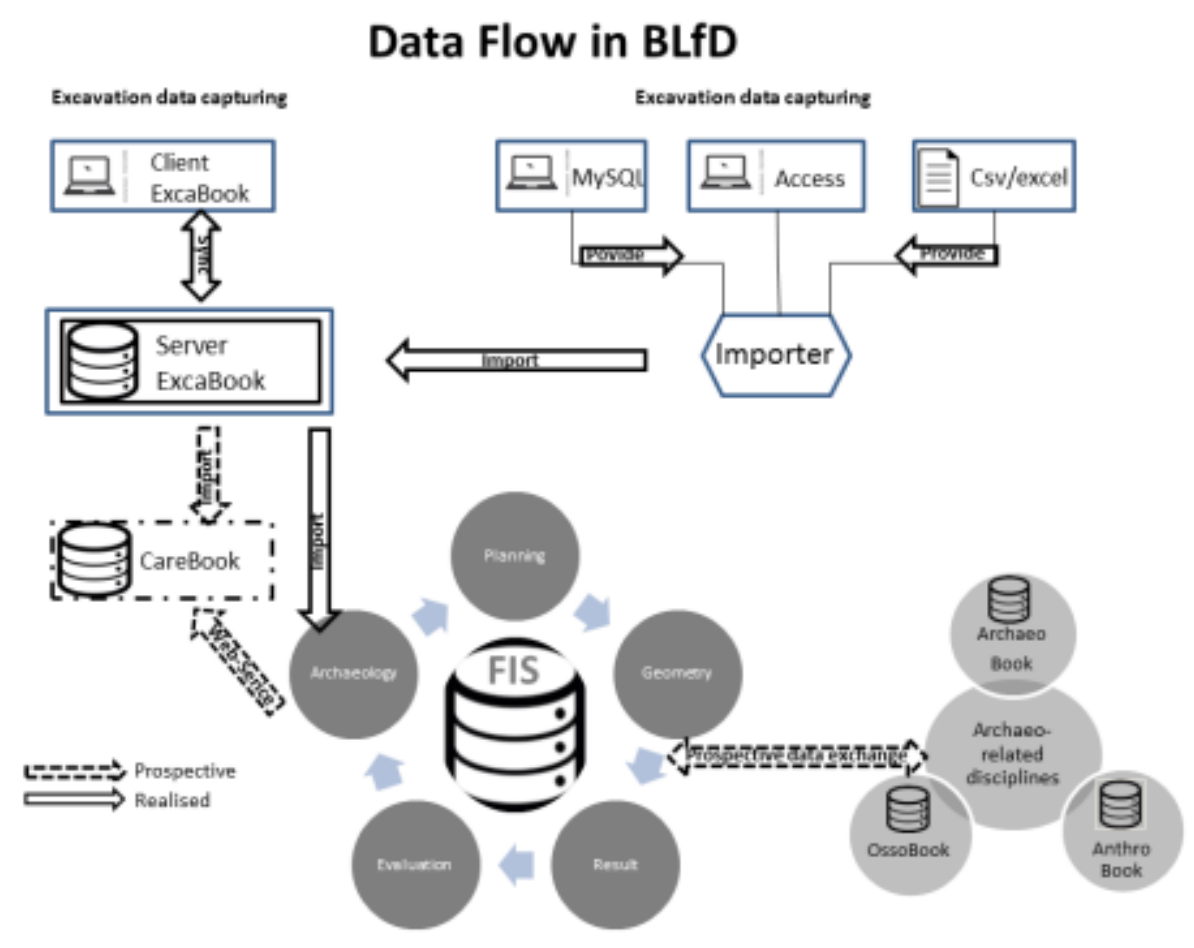

Figure 5: Data flow within the BLfD

Kaltenthaler, D. 2018 Supporting the Workflow of Archaeo-related Sciences by Providing Storage, Sharing, Analysis, and Retrieval Methods, Dissertation zur Erlangung des Doktorgrades an der Fakultät für Mathematik, Informatik und Statistik der LudwigMaximilians-Universität München; 15.02.2018. https://d-nb.info/1161341994/34

Kaltenthaler, D., Lohrer, J., Kröger, P., van der Meijden, C., Wanke, T., Sassen, I., Jantos, S., Rahm, A., Wanninger, R., Haberstroh, J. and Sommer, S. 2020 ExcaBook v20.3, Software, München. http://xbook.vetmed.uni-muenchen.de/ 